ISSN: 1813-162X (Print) ; 2312-7589 (Online)
available online at: http://www.tj-es.com Tikrit Journal of Engineering Sciences

\section{Rodhan Abdullah Salih \\ Idan I. Ghdhban \\ AbdulRazaq Khader Abdul Wahid*}

Technical Institute Hawijah Northern Technical University Kirkuk, Hawijah

Iraq

\title{
Evaluation of a Number of Water Treatment Plants in Kirkuk Governorate using the Water Quality Index
}

\author{
A B S T R A C T
}

A study was conducted on sixteen water purification plants in Kirkuk governorate to evaluate the treatment of water in them, where physical and chemical tests were conducted for raw water and treated water for a period of (6) months from December until May. Temperature, turbidity, pH, Total Dissolved Solid (TDS), Electric Conductivity (EC), alkali, Total Hardness (TH) and calcium $\left(\mathrm{Ca}^{+2}\right)$ were measured. Water quality index Canadian method (CCME) was used to classify raw water quality and treated water. The results showed that the raw water for all stations was classified as category (4) (bad) during the study period. The treated water was different for the treatment plants. Two of the treatment plants recorded good efficiency in water treatment (AL-Shallalah plant and Sin AL-Thiban) the treated water remained in category (2) (good). While the water quality of ALMosanaa plant indicated that there was a problem in the treatment of water in this plant, the treated water remained in category (4) bad during the study period. Water quality index fluctuated for other plants during the study period. The study also showed that alkali values of all stations were higher than the allowable limit for raw water and treated water.

(c) 2018 TJES, College of Engineering, Tikrit University

DOI: http://dx.doi.org/10.25130/tjes.25.1.08

تقييم عدد من محطات تصفية المياه الخام في محافظة كركوك باستخدام مؤشر نوعية الماء

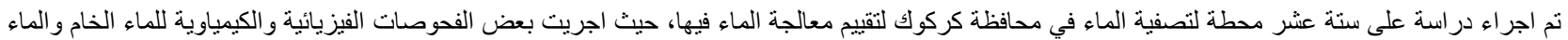

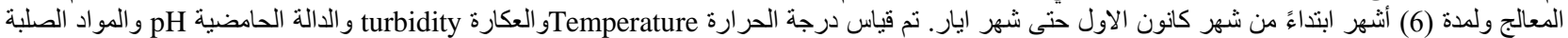

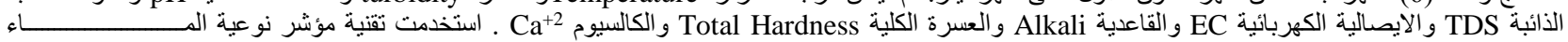

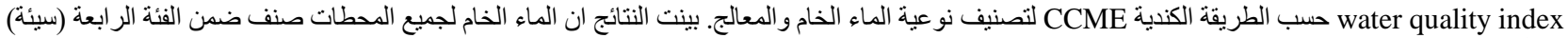

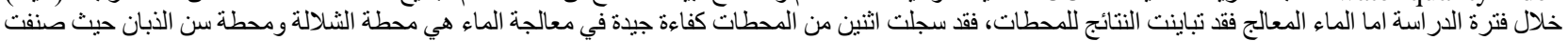

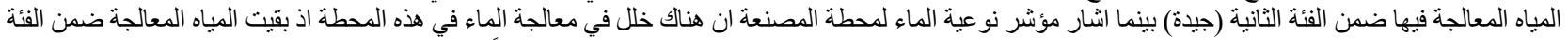

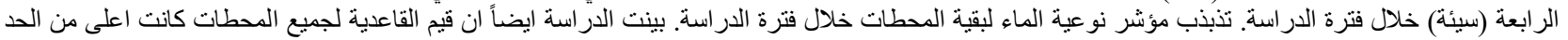

المسموح به للماء الخام و المعالج.

\section{INTRODUCTION}

It is known that the goal of any water treatment plant is to produce safe water for drinking and tasteful and suitable for domestic use [1]. The water treatment is carried out in the form of sedimentation and filtration units to remove the impurities. Water is a quick way to spread many diseases and parasites. The water medium is an important part of the life cycle of some pathogens. The WHO reports that $80 \%$ Human rights in developing countries are related to water pollution [2]. The water quality depends on its physical, chemical and biological properties. The water contains impurities in addition to 
dissolved materials. These impurities are often removed or reduced to certain limits to make the water potable [3]. Several studies have been conducted to evaluate the efficiency of water treatment plants in different regions in order to determine the suitability and need of these plants for the maintenance and control of water production. Several methods were used to evaluate the efficiency of the filtration plants. The water quality index is one of the methods used to evaluate the quality of water in the water treatment plant and other water sources, where scientists and water quality experts have developed the water quality index (WQI). This indicator is considered the preferred scientific method because it uses many qualitative variables Water and its formulation in numerical expression includes the integrated effect of these variables on the quality of water and has an effective role in the process of control of water quality and strategic management so that the classification of water qualitatively for various activities within a specific category in a simple scientific and useful [4]. The water quality index was first used in 1965 by Horton and was developed by several researchers later. Abdul-Rahman and Ahmed [5] used the Canadian method of calculating the water quality index (CCME 2001) using this method to study a number of water treatment plants in Baghdad [6]. WQI (Waleed et al.) was used to study a number of water treatment plants in Tikrit. Zainab used the same method to evaluate the efficiency of a number of water treatment plants in Baghdad. Al-Hadithi [4] used the water quality index to assess water quality for wells in Al-Qaim city, Anbar province Al-Alwani and Yassin [7] studied the validity of surface and groundwater for consumption in the city of Zintan Libya and using the method of water quality index [8]. Muthanna used the Canadian method to calculate the water quality index for a number of water treatment plants in Salah al-Din Governorate. AL-Bassam et al. [9] studied groundwater quality in Jingju province, China using water quality index [10]. Al-Badran calculated the water quality of some of water treatment plants in the province of Basra [11].

The current study aims to evaluate a number of water treatment plants in the western Kirkuk area by using the water quality index based on the measurement of a number of physical and chemical properties of the raw water and the treatment of these plants based on the Iraqi standards for drinking water and comparing the results with the Canadian classification of water quality according to Table 1.

Table 1

Canadian classification for water quality index (CCME)[6].

\begin{tabular}{lll}
\hline $\begin{array}{l}\text { Water } \\
\text { quality class }\end{array}$ & Category & WQI \\
\hline Excellent & 1 & $95-100$ \\
Good & 2 & $80-94$ \\
Moderate & 3 & $65-79$ \\
Bad & 4 & $45-64$ \\
Very bad & 5 & $0-44$ \\
\hline
\end{tabular}

\section{PRACTICAL PART}

Sixteen water treatment plants were selected in different areas west of Kirkuk Governorate. As shown in Table 2, samples were taken for raw water from the plants intakes. The treated water samples were taken from the distributed network. Plastic bottles were used to store the samples. Examine each month for a period of (6) months starting from December until May according to standard methods [12]. Temperature, turbidity, pH, Total Dissolved Solid, Electrical conductivity, Alkali and Total Hardness were examined) and calcium, and used Iraqi standard specifications for drinking water to compare the results $[1,13]$.

\subsection{Water Quality Index Calculation}

The Canadian method was used to calculate the WQI water quality index $[4,6]$. The method computes three parameters: scope F1, frequency F2, and amplitude F3. The first factor F1 is calculated from the equation:

$F 1=\frac{\text { No. of failed parameters }}{\text { total parmeters }} \times 100$

The second factor F2 is then calculated from the equation:

$F 2=\frac{\text { No. of failed tests }}{\text { total tests }} \times 100$

Table 2

Water treatment plants names and site.

\begin{tabular}{|c|c|c|}
\hline No. & Plant Name & GPS \\
\hline 1 & Al-Faris & $\begin{array}{l}\mathrm{E}=43^{\circ} 40^{\prime} 36^{\prime \prime}, \\
\mathrm{N}=35^{\circ} 21^{\prime} 02^{\prime \prime}\end{array}$ \\
\hline 2 & Al-Gasia & $\begin{array}{l}\mathrm{E}=43^{\circ} 45^{\prime} 40^{\prime \prime}, \\
\mathrm{N}=35^{\circ} 23^{\prime} 15^{\prime \prime}\end{array}$ \\
\hline 3 & Al- Kadhimiya & $\begin{array}{l}\mathrm{E}=43^{0} 46^{\prime} 20^{\prime \prime}, \\
\mathrm{N}=35^{\circ} 22^{\prime} 55^{\prime \prime}\end{array}$ \\
\hline 4 & Arisha & $\begin{array}{l}\mathrm{E}=43^{\circ} 4830^{\prime \prime}, \\
\mathrm{N}=35^{\circ} 27^{\prime} 54^{\prime \prime}\end{array}$ \\
\hline 5 & Al-Musanaa & $\begin{array}{l}\mathrm{E}=43^{\circ} 46^{\prime} 32^{\prime \prime}, \\
\mathrm{N}=35^{\circ} 22^{\prime} 28^{\prime \prime}\end{array}$ \\
\hline 6 & Tel Hussein & $\begin{array}{l}\mathrm{E}=43^{\circ} 43^{\prime} 50^{\prime \prime}, \\
\mathrm{N}=35^{\circ} 20^{\prime} 14^{\prime \prime}\end{array}$ \\
\hline 7 & Hoth 6 & $\begin{array}{l}\mathrm{E}=43^{\circ} 38^{\prime} 24^{\prime \prime}, \\
\mathrm{N}=35^{\circ} 18^{\prime} 00^{\prime \prime}\end{array}$ \\
\hline 8 & AL Basal & $\begin{array}{l}E=43^{\circ} 39^{\prime} 07^{\prime \prime}, \\
N=35^{\circ} 17^{\prime} 53^{\prime \prime}\end{array}$ \\
\hline 9 & Tal Ali & $\begin{array}{l}\mathrm{E}=43^{\circ} 38^{\prime} 35^{\prime \prime}, \\
\mathrm{N}=35^{\circ} 22^{\prime} 02^{\prime \prime}\end{array}$ \\
\hline 10 & Al-Shalalah & $\begin{array}{l}\mathrm{E}=43^{\circ} 43^{\prime} 16^{\prime \prime}, \\
\mathrm{N}=35^{\circ} 23^{\prime} 07^{\prime \prime}\end{array}$ \\
\hline 11 & Sin Althiban & $\begin{array}{l}\mathrm{E}=43^{\circ} 51^{\prime} 25^{\prime \prime}, \\
\mathrm{N}=35^{\circ} 30^{\prime} 21^{\prime \prime}\end{array}$ \\
\hline 12 & Al Shajarah & $\begin{array}{l}\mathrm{E}=43^{\circ} 24^{\prime} 49^{\prime \prime} \\
\mathrm{N}=35^{\circ} 12^{\prime} 17^{\prime \prime}\end{array}$ \\
\hline 13 & Gharib & $\begin{array}{l}\mathrm{E}=43^{\circ} 29^{\prime} 06^{\prime \prime}, \\
\mathrm{N}=35^{\circ} 14^{\prime} 30^{\prime \prime}\end{array}$ \\
\hline 14 & Al Tarqia & $\begin{array}{l}\mathrm{E}=43^{\circ} 34^{\prime} 01^{\prime \prime}, \\
\mathrm{N}=35^{\circ} 15^{\prime} 02^{\prime \prime}\end{array}$ \\
\hline 15 & Abbasid & $\begin{array}{l}\mathrm{E}=43^{\circ} 35^{\prime} 47^{\prime \prime}, \\
\mathrm{N}=35^{\circ} 16^{\prime} 32^{\prime \prime}\end{array}$ \\
\hline 16 & Abu al-Jess & $\begin{array}{l}E=43^{\circ} 50^{\prime} 50^{\prime \prime}, \\
N=35^{\circ} 17^{\prime} 46^{\prime \prime}\end{array}$ \\
\hline
\end{tabular}

The third factor F3 is calculated in three stages:

-Calculate the deviation of each failed test from the equation:

Excursion $i=\frac{\text { failed test value }}{\text { objective }}-1$ 
- Calculate the normalized state of exclusions from the equation:

$n s e=\frac{\sum \text { excursios }}{\text { No. of tests }}$

- The third factor F3 is calculated from the equation:

$$
F 3=\frac{\text { nse }}{(0.01 \times \mathrm{nse}+0.01)}
$$

The results of the tests were presented in Tables 3-18

After finding the three factors, the water quality index is calculated from the equation:

$W Q I=100-\frac{\sqrt{F 1^{2}+F 2^{2}+F 3^{2}}}{1.732}$

The water quality index for each station was calculated based on the laboratory results using the equations above and then comparing the results obtained for each station with the Table 1 for the category representing the quality of the sample examined according to the table and the diagram of the change in the water quality throughout the study period using the excel program Variance during this period for raw and treated water.

\section{RESULTS AND DISCUSSION}

Fig. 1 represents the water quality index (WQI) of AlFaris water treatment plant. The results showed that the raw water was in category 4 (bad) according to the classification (CCME 2001) shown in Table 1 The second category (good) for the first three months of the study, but in the next three months was the classification of water treated in the third category (moderate) and Table 3, which shows the results of tests for this plant shows that the electric conductivity (EC) in the last three months was higher than the specifications, The decline in the quality of treated water from the second category to the third category in addition to that alkali was higher than the standard for all months of the study.

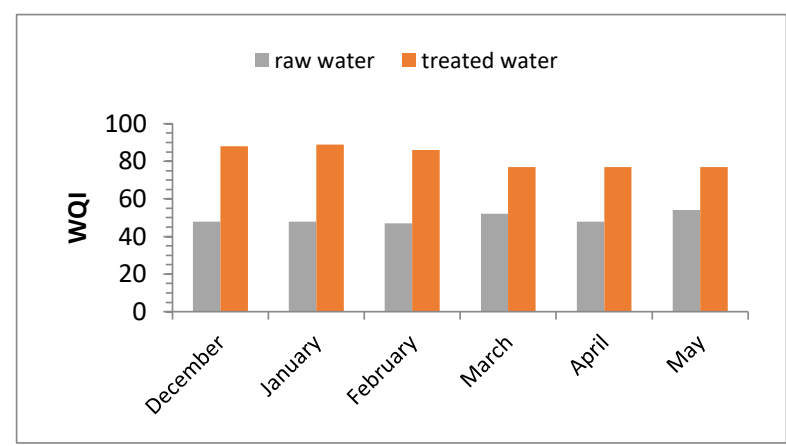

Fig. 1. WQI for Al-Faris water treatment plant.

Fig. 2 shows the water quality of Al-Gasia water treatment plant. In this Figure, raw water was found to be in Category 4 (bad) because the turbidity exceeded the limits allowed in the specification as shown in Table (4) The quality of the water was in the second category (good) during the month of December and May, but in the other four months, the water treated in this plant within the third category (moderate).

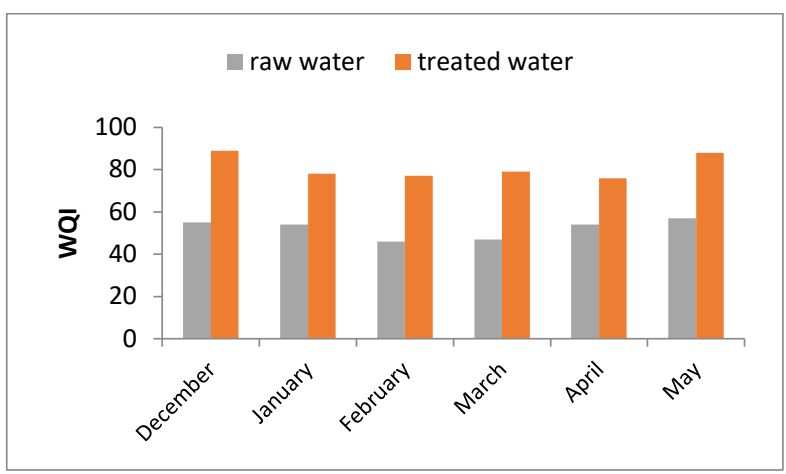

Fig. 2. Water quality index of Al-Gasia water treatment plant.

The water quality index (WQI) of Al- Kadhimiya water treatment plant shown in Fig. 3 for raw water was classified as Category 5 (very bad) for December and Jan., as turbidity, EC and alkali exceeded the allowable limits in Table 5 and became in the fourth category (bad) for the next four months. After treatment, the water was classified into the third category (moderate) during the study period.

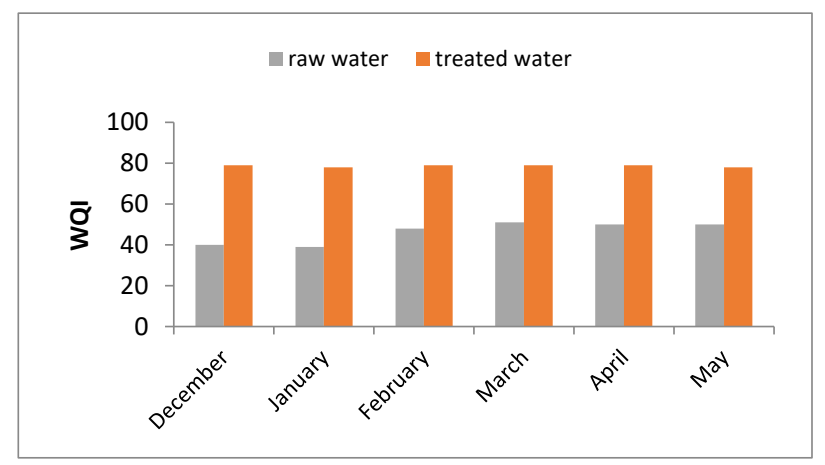

Fig. 3. Water quality index of Kadhimiya water treatment plant.

(Arisha) water treatment plant experienced fluctuation in the water quality index during the study period as shown in Fig. 4. The raw water was classified in the fourth category (bad) for the first and second months of the study and was classified in the third category (moderate) Subsequent, either treated water has fluctuated between good and moderate and bad as shown in Table 6 .

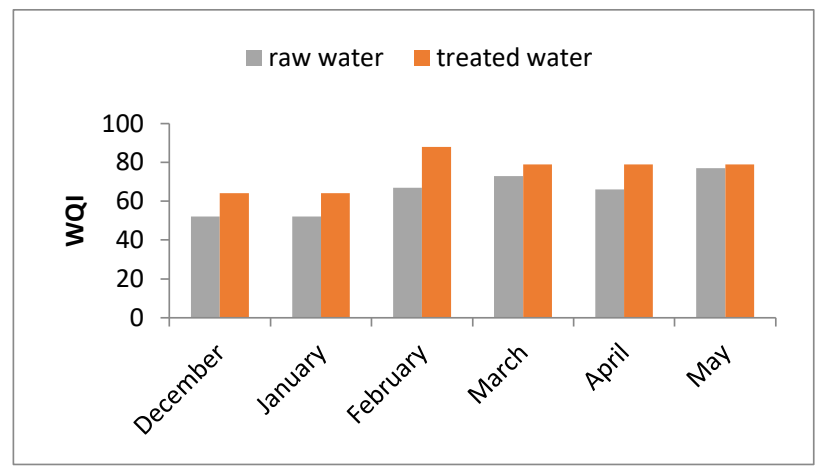

Fig. 4. Water quality index of Arisha water treatment plant.

Al-Musanaa water treatment plant results showed a defect in water treatment as shown in Fig. 5. Raw water in category (4) was classified as bad during the study period, while the classification of treated water was not changed after treatment as in Table 7. 


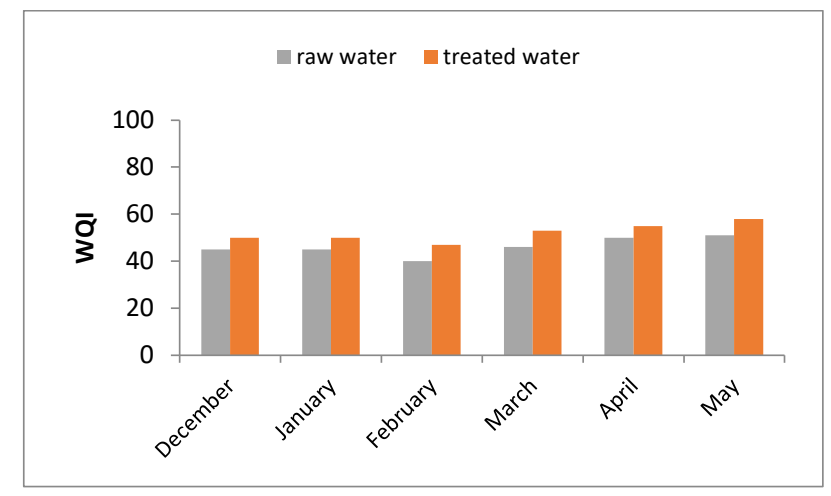

Fig. 5. Water quality index of Al-Musanaa water treatment plant.

Fig. 6 shows the water quality of (Tel Hussein) water treatment plant showing the results of its tests in Table 8, where the raw water was classified as category 4 (bad) except for December was in the second category (good), the treated water was classified in category III (moderate) for all months of the study.

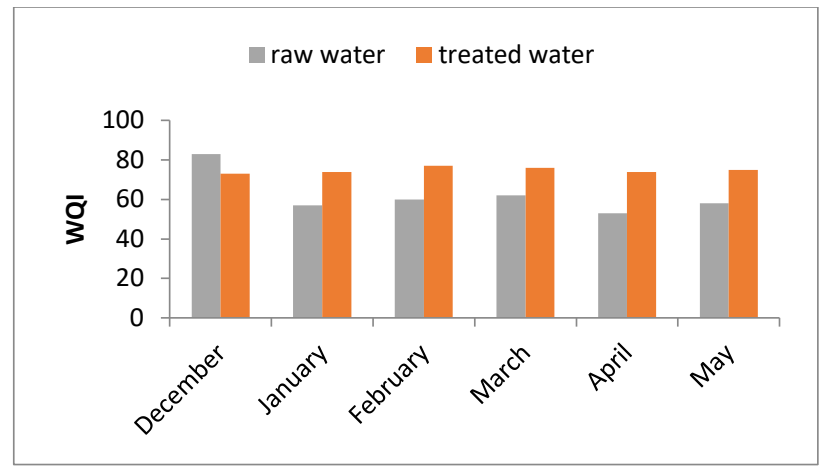

Fig. 6. Water quality index of Tel Hussein water treatment plant.

The results shown in Fig. 7 showed that there was a defect in the treatment of water for the month of December and Jan. in (Hoth 6) water treatment plant where the raw and treated water in the fourth category (bad) That the turbidity values remained above the allowable limit after treatment, indicating a defect in the sedimentation and filtration units.

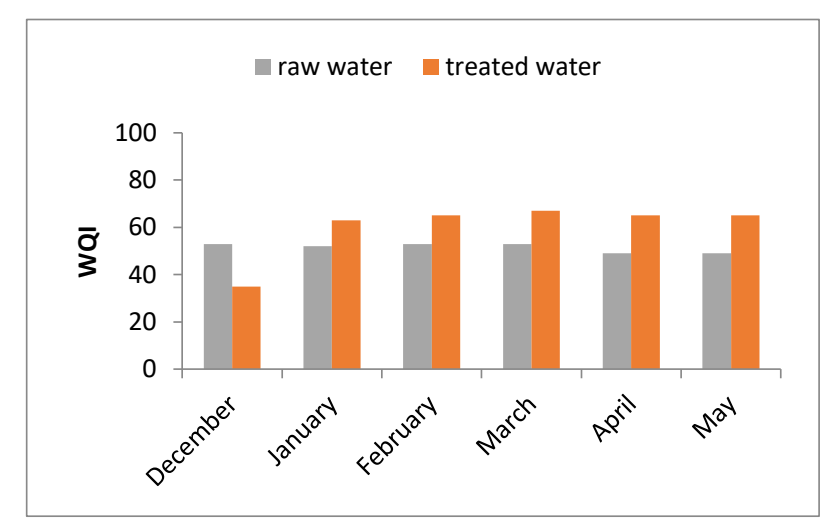

Fig. 7. Water quality index of Hoth 6 water treatment plant.

A raw water for $\mathrm{AL}$ Basal water treatment plant shown in Fig. 8 was between the third category (moderate) in December and April and the fourth category for the rest of the study months. The treated water was in the third category (moderate) except in May. (Good) as is evident from Table 10.

Fig. 9 represents the water quality index (WQI) of Tal Ali water treatment plant. The results showed that the raw water was in the fourth category (bad). The treated water showed the results because the water quality did not improve in the first two months, while the indicator improved for the next four months to the third category moderate) as is evident in the Table 11.

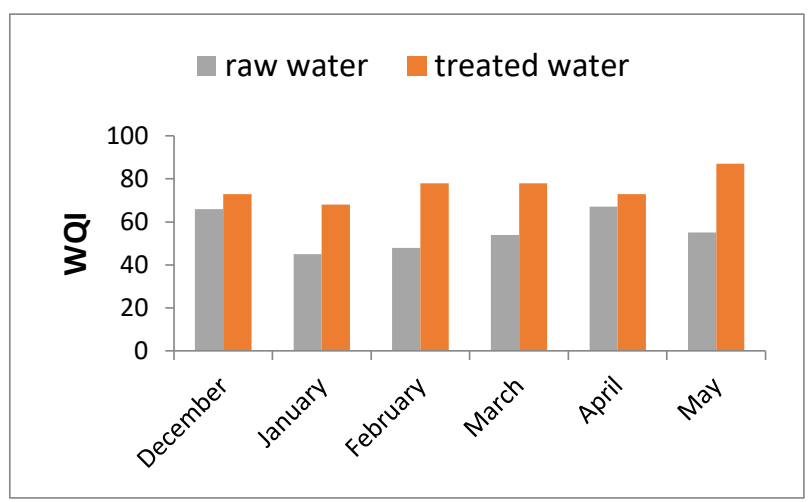

Fig. 8. Water quality index AL Basal water treatment plant.

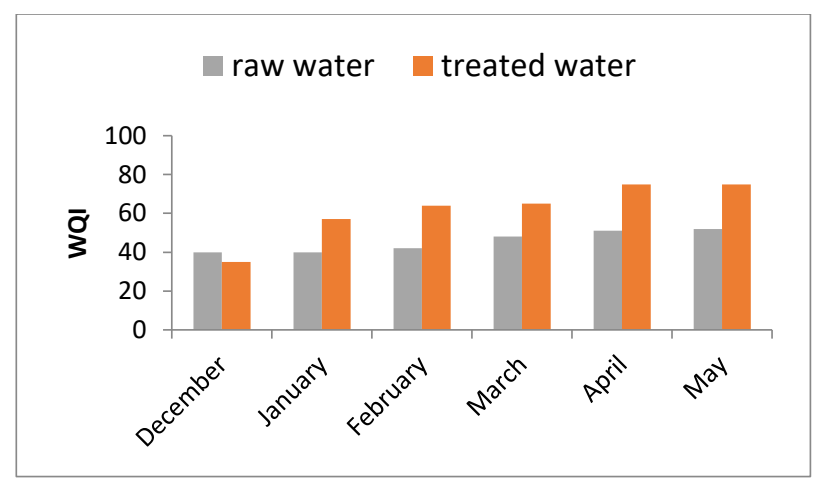

Fig. 9. Water quality index Tal Ali water treatment plant.

in Al-Shalalah and Sin Althiban water treatment plants The results of the water quality index showed good water treatment efficiency as shown in Figs 10 and 11 respectively, as the raw water quality index of the two plants in category 4 (bad) improved after treatment to the second category for both plants and for all study months as shown in Tables 12 and 13.

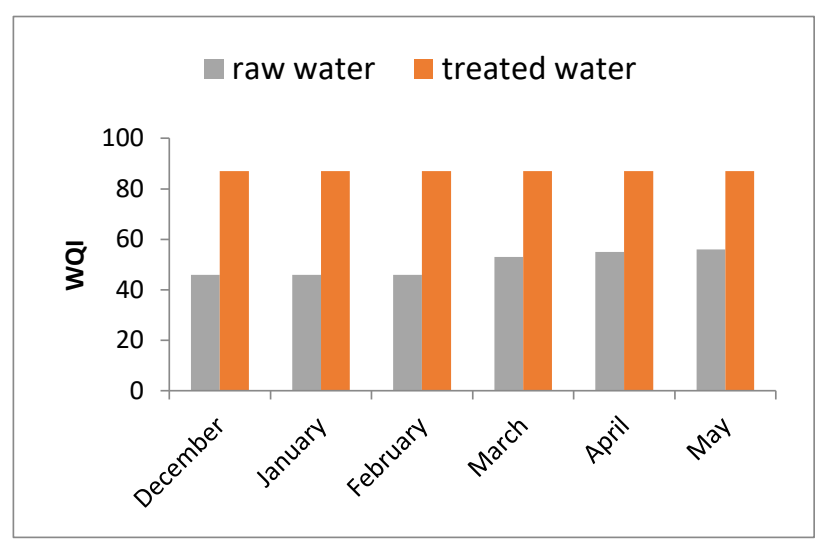

Fig. 10. Water quality index Al-Shalalah water treatment plant. 
Table 3

Chemical and physical tests for Al-Faris water treatment plant.

\begin{tabular}{|c|c|c|c|c|c|c|c|c|c|c|c|c|c|c|c|c|c|}
\hline \multicolumn{2}{|c|}{ WQI } & \multicolumn{2}{|c|}{$\mathbf{C a}^{+2}$} & \multicolumn{2}{|c|}{ TH } & \multicolumn{2}{|c|}{ Alkali } & \multicolumn{2}{|c|}{ EC } & \multicolumn{2}{|c|}{ TDS } & \multicolumn{2}{|c|}{$\mathbf{P H}$} & \multicolumn{2}{|c|}{ Turbidity } & \multirow[b]{2}{*}{ 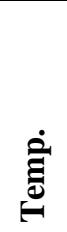 } & \multirow[b]{2}{*}{ 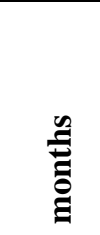 } \\
\hline 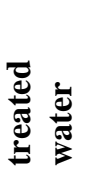 & 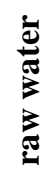 & 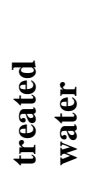 & 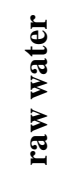 & 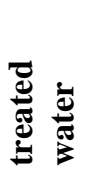 & 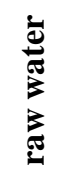 & 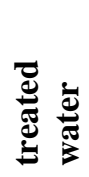 & 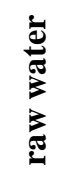 & 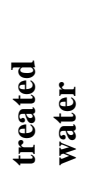 & 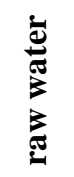 & 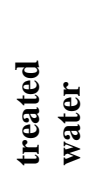 & 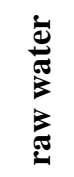 & 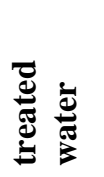 & 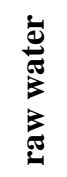 & 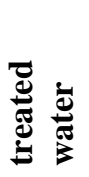 & 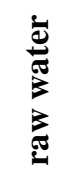 & & \\
\hline 88 & 48 & 0.75 & 0.7 & 40 & 98 & 389 & 579 & 460 & 438 & 334 & 315 & 7.5 & 8.3 & 2.9 & 181 & 10 & Dec. \\
\hline 89 & 48 & 0.9 & 0.6 & 41 & 98 & 280 & 580 & 461 & 438 & 330 & 314 & 8.52 & 8.4 & 2.9 & 181 & 9.4 & Jan. \\
\hline 86 & 47 & 0.9 & 0.5 & 40 & 49 & 514 & 801 & 470 & 442 & 344 & 314 & 7.9 & 8.3 & 3 & 191 & 9.0 & Feb. \\
\hline 77 & 52 & 1.1 & 1.5 & 31 & 60 & 510 & 709 & 510 & 500 & 383 & 390 & 8.2 & 8.5 & 2 & 117 & 20 & March \\
\hline 77 & 48 & 1.9 & 2.1 & 19 & 81 & 560 & 614 & 503 & 501 & 399 & 393 & 7.9 & 7.9 & 1.8 & 100 & 20 & April \\
\hline \multirow[t]{2}{*}{77} & 54 & 0.9 & 2 & 20 & 80 & 550 & 615 & 507 & 500 & 390 & 385 & 8.1 & 8 & 1.1 & 96 & 20 & May \\
\hline & & \multicolumn{2}{|c|}{$\begin{array}{c}200-125 \\
\mathrm{mg} / \mathrm{l}\end{array}$} & \multicolumn{2}{|c|}{$500 \mathrm{mg} / 1$} & \multicolumn{2}{|c|}{$200 \mathrm{mg} / \mathrm{l}$} & \multicolumn{2}{|c|}{$\begin{array}{c}500 \\
\text { Mmohs/cm }\end{array}$} & \multicolumn{2}{|c|}{$1000 \mathrm{mg} / \mathrm{l}$} & \multicolumn{2}{|c|}{$8.5-6.5$} & \multicolumn{2}{|c|}{$5 \mathrm{NTU}$} & 25 & S.S \\
\hline
\end{tabular}

Table 4

Chemical and physical tests for Al-Gasia water treatment plant.

\begin{tabular}{|c|c|c|c|c|c|c|c|c|c|c|c|c|c|c|c|c|c|}
\hline \multicolumn{2}{|c|}{ WQI } & \multicolumn{2}{|c|}{$\mathrm{Ca}^{+2}$} & \multicolumn{2}{|c|}{ TH } & \multicolumn{2}{|c|}{ Alkali } & \multicolumn{2}{|c|}{ EC } & \multicolumn{2}{|c|}{ TDS } & \multicolumn{2}{|c|}{ PH } & \multicolumn{2}{|c|}{ Turbidity } & \multirow[b]{2}{*}{$\stackrel{\dot{\vec{g}}}{\ddot{\Xi}}$} & \multirow[b]{2}{*}{$\begin{array}{l}\mathscr{n} \\
\stackrel{\Xi}{\Xi}\end{array}$} \\
\hline 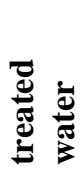 & 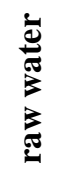 & 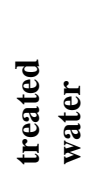 & 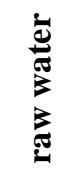 & 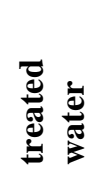 & 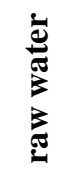 & 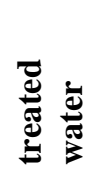 & 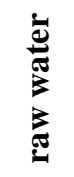 & 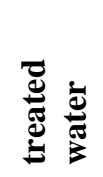 & 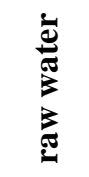 & 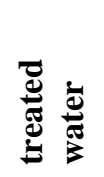 & 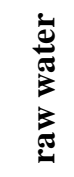 & 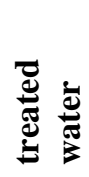 & 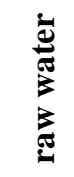 & 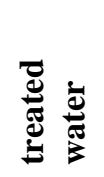 & 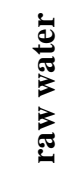 & & \\
\hline 89 & 55 & 0.99 & 0.8 & 59 & 80 & 650 & 825 & 470 & 450 & 330 & 310 & 8 & 7.8 & 5 & 80 & 10 & Dec. \\
\hline 78 & 54 & 0.99 & 0.7 & 62 & 83 & 640 & 830 & 470 & 443 & 335 & 320 & 8.2 & 7.6 & 5.3 & 80.2 & 9.4 & Jan. \\
\hline 77 & 46 & 0.53 & 1.2 & 31 & 91 & 679 & 710 & 525 & 502 & 366 & 342 & 8.1 & 7.8 & 4.1 & 124 & 9.0 & Feb. \\
\hline 79 & 47 & 1.3 & 1.9 & 33 & 70 & 519 & 790 & 539 & 503 & 362 & 315 & 7.5 & 7.3 & 3.9 & 99 & 20 & March \\
\hline 76 & 54 & 1.8 & 2 & 21 & 50 & 760 & 781 & 580 & 479 & 334 & 312 & 8 & 7.8 & 3 & 88 & 20 & April \\
\hline \multirow[t]{2}{*}{88} & 57 & 1.6 & 1 & 22 & 49 & 760 & 780 & 493 & 480 & 333 & 312 & 8 & 7.6 & 2.5 & 70 & 20 & May \\
\hline & & \multicolumn{2}{|c|}{$\begin{array}{c}200-125 \\
\mathrm{mg} / \mathrm{l}\end{array}$} & \multicolumn{2}{|c|}{$500 \mathrm{mg} / \mathrm{l}$} & \multicolumn{2}{|c|}{$200 \mathrm{mg} / \mathrm{l}$} & \multicolumn{2}{|c|}{$\begin{array}{c}500 \\
\mathrm{Mmohs} / \mathrm{cm}\end{array}$} & \multicolumn{2}{|c|}{$1000 \mathrm{mg} / \mathrm{l}$} & \multicolumn{2}{|c|}{$8.5-6.5$} & \multicolumn{2}{|c|}{$5 \mathrm{NTU}$} & 25 & I.S.S \\
\hline
\end{tabular}

Table 5

Chemical and physical tests for Al- Kadhimiya water treatment plant.

\begin{tabular}{|c|c|c|c|c|c|c|c|c|c|c|c|c|c|c|c|c|c|}
\hline \multicolumn{2}{|c|}{ WQI } & \multicolumn{2}{|c|}{$\mathrm{Ca}^{+2}$} & \multicolumn{2}{|c|}{ TH } & \multicolumn{2}{|c|}{ Alkali } & \multicolumn{2}{|c|}{ EC } & \multicolumn{2}{|c|}{ TDS } & \multicolumn{2}{|c|}{ PH } & \multicolumn{2}{|c|}{ Turbidity } & \multirow[b]{2}{*}{ 苞 } & \multirow[b]{2}{*}{ 总 } \\
\hline 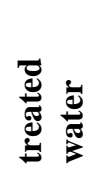 & 离 & 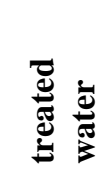 & 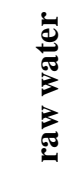 & 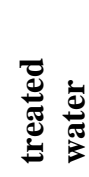 & 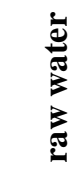 & 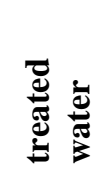 & 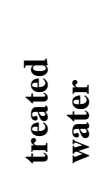 & 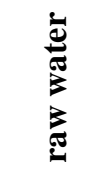 & 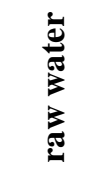 & 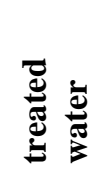 & 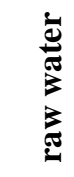 & 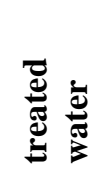 & 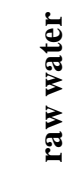 & 曾 & 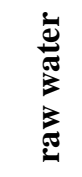 & & \\
\hline 79 & 40 & 0.99 & 0.9 & 25 & 38 & 551 & 860 & 551 & 530 & 400 & 379 & 7.2 & 7.7 & 0.5 & 250 & 10 & Dec. \\
\hline 78 & 39 & 0.94 & 0.85 & 25 & 38 & 550 & 870 & 558 & 530 & 403 & 379 & 7.2 & 7.7 & 0.5 & 302 & 9.4 & \\
\hline 79 & 48 & 1.1 & 1 & 56 & 69 & 623 & 760 & 581 & 504 & 410 & 390 & 7.3 & 7.9 & 0.9 & 400 & 9.0 & Feb. \\
\hline 79 & 51 & 1.3 & 1.5 & 17 & 53 & 401 & 509 & 586 & 515 & 455 & 399 & 8 & 7.8 & 1 & 103 & 20 & March \\
\hline 79 & 50 & 1.9 & 2.5 & 23 & 90 & 510 & 470 & 589 & 573 & 410 & 385 & 7.9 & 7.5 & 0.8 & 77 & 20 & April \\
\hline \multirow[t]{2}{*}{78} & 50 & 1.4 & 1.5 & 22 & 93 & 552 & 860 & 540 & 533 & 420 & 385 & 7.9 & 7.6 & 0.9 & 70 & 20 & May \\
\hline & & $\begin{array}{l}200-1 \\
\mathrm{mg} / \mathrm{l}\end{array}$ & & 500 & $\mathrm{ng} / \mathrm{l}$ & 200 & & $\begin{array}{l}500 \\
\text { Mmo }\end{array}$ & $\mathrm{hs} / \mathrm{cm}$ & 10 & & 8.5 & & $5 \mathrm{NT}$ & & 25 & S.S \\
\hline
\end{tabular}


Table 6

Chemical and physical tests for Arisha water treatment plant.

\begin{tabular}{|c|c|c|c|c|c|c|c|c|c|c|c|c|c|c|c|c|c|}
\hline \multicolumn{2}{|c|}{ WQI } & \multicolumn{2}{|c|}{$\mathrm{Ca}^{+2}$} & \multicolumn{2}{|c|}{ TH } & \multicolumn{2}{|c|}{ Alkali } & \multicolumn{2}{|c|}{ EC } & \multicolumn{2}{|c|}{ TDS } & \multicolumn{2}{|c|}{$\mathbf{P H}$} & \multicolumn{2}{|c|}{ Turbidity } & \multirow[b]{2}{*}{$\stackrel{\dot{\Xi}}{\stackrel{\Xi}{\rightleftarrows}}$} & \multirow[b]{2}{*}{$\begin{array}{l}\text { E } \\
\stackrel{\Xi}{\Xi}\end{array}$} \\
\hline 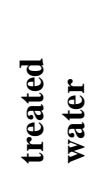 & 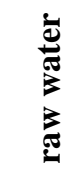 & 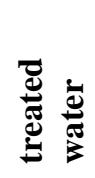 & 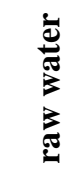 & 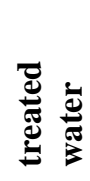 & 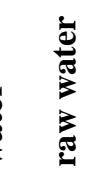 & 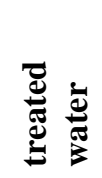 & 㐫 & 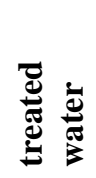 & 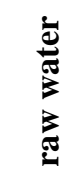 & 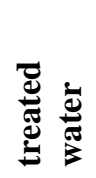 & 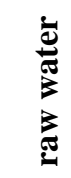 & 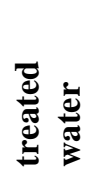 & 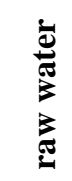 & 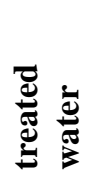 & 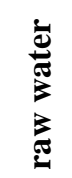 & & \\
\hline 64 & 52 & 0.97 & 0.7 & 63 & 95 & 179 & 365 & 455 & 425 & 319 & 306 & 7.9 & 8.5 & 62 & 115 & 10 & Dec. \\
\hline 64 & 52 & 0.97 & 0.7 & 62 & 95 & 0 & 370 & 455 & 430 & 319 & 306 & 7.9 & & 62 & 116 & 9.4 & \\
\hline 88 & 67 & 0.5 & 0.9 & 62 & 95 & 182 & 300 & 401 & 399 & 352 & 346 & 8.5 & 8.5 & 8.5 & 35 & 9.0 & Feb. \\
\hline 79 & 73 & 0.9 & 1.1 & 21 & 93 & 193 & 400 & 400 & 415 & 489 & 400 & 8.1 & 8.2 & 8.8 & 16 & 20 & March \\
\hline 79 & 66 & 1.9 & 2.5 & 31 & 85 & 200 & 335 & 422 & 409 & 405 & 386 & 8 & 8 & 8 & 14 & 20 & April \\
\hline \multirow[t]{2}{*}{79} & 77 & 2.1 & 2.8 & 22 & 80 & 160 & 330 & 430 & 410 & 399 & 380 & 7.9 & 7.9 & 7 & 10 & 20 & May \\
\hline & & $\begin{array}{l}200-12 \\
\mathrm{mg} / \mathrm{l}\end{array}$ & & 500 & $\mathrm{mg} / \mathrm{l}$ & 200 & & $\begin{array}{l}500 \\
\text { Mmoh }\end{array}$ & $\mathrm{s} / \mathrm{cm}$ & 00 & & 8 & & 1010 & & 25 & I.S.S \\
\hline
\end{tabular}

Table 7

Chemical and physical tests for Al-Musanaa water treatment plant.

\begin{tabular}{|c|c|c|c|c|c|c|c|c|c|c|c|c|c|c|c|c|c|}
\hline \multicolumn{2}{|c|}{ WQI } & \multicolumn{2}{|c|}{$\mathrm{Ca}^{+2}$} & \multicolumn{2}{|c|}{ TH } & \multicolumn{2}{|c|}{ Alkali } & \multicolumn{2}{|c|}{ ec } & \multicolumn{2}{|c|}{ TDS } & \multicolumn{2}{|c|}{$\mathbf{P h}$} & \multicolumn{2}{|c|}{ turbidity } & \multirow[b]{2}{*}{$\stackrel{\dot{\vec{g}}}{\ddot{\theta}}$} & \multirow[b]{2}{*}{ } \\
\hline 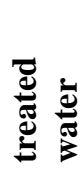 & 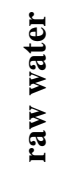 & 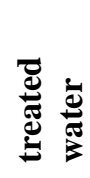 & 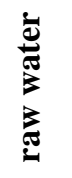 & 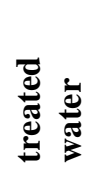 & 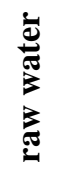 & 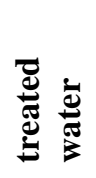 & 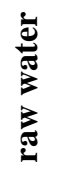 & 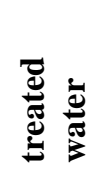 & 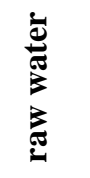 & 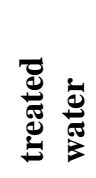 & 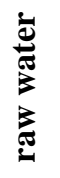 & 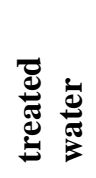 & 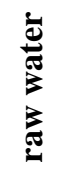 & 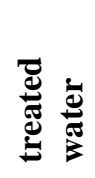 & 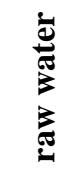 & & \\
\hline 50 & 45 & 1.05 & 0.94 & 96 & 115 & 508 & 735 & 487 & 458 & 349 & 329 & 7.36 & 7.47 & 140 & 285 & 10 & Dec. \\
\hline 50 & 45 & 1.01 & 0.90 & 98 & 118 & 510 & 750 & 496 & 460 & 354 & 331 & 7.38 & 7.46 & 144 & 280 & 9.4 & Jan. \\
\hline 47 & 40 & 1.33 & 1.70 & 49.3 & 59.2 & 793 & 910 & 752 & 690 & 570 & 553 & 7.2 & 7.9 & 101 & 268 & 9.0 & Feb. \\
\hline 53 & 46 & 1 & 0.91 & 21 & 64 & 600 & 803 & 640 & 550 & 494 & 492 & 8 & 7.8 & 55 & 113 & 20 & March \\
\hline 55 & 50 & 1.20 & 0.74 & 17 & 58 & 536 & 750 & 520 & 490 & 743 & 886 & 7.5 & 7.8 & 49 & 130 & 20 & April \\
\hline \multirow[t]{2}{*}{58} & 51 & 1.25 & 0.77 & 15 & 57 & 547 & 785 & 520 & 488 & 742 & 880 & 7.5 & 7.8 & 35 & 125 & 20 & May \\
\hline & & \multicolumn{2}{|c|}{$\begin{array}{l}200-125 \\
\mathrm{mg} / \mathrm{l}\end{array}$} & \multicolumn{2}{|c|}{$500 \mathrm{mg} / \mathrm{l}$} & \multicolumn{2}{|c|}{$200 \mathrm{mg} / 1$} & \multicolumn{2}{|c|}{$\begin{array}{l}\text { 500Mmohs/ } \\
\mathrm{cm}\end{array}$} & \multicolumn{2}{|c|}{$1000 \mathrm{mg} / \mathrm{l}$} & \multicolumn{2}{|c|}{$8.5-6.5$} & \multicolumn{2}{|c|}{$5 \mathrm{NTU}$} & 25 & I.S.S \\
\hline
\end{tabular}

Table 8

Chemical and physical tests for Tel Hussein water treatment plant.

\begin{tabular}{|c|c|c|c|c|c|c|c|c|c|c|c|c|c|c|c|c|c|}
\hline \multicolumn{2}{|c|}{ WQI } & \multicolumn{2}{|c|}{$\mathrm{Ca}^{+2}$} & \multicolumn{2}{|c|}{ TH } & \multicolumn{2}{|c|}{ Alkali } & \multicolumn{2}{|c|}{ Ec } & \multicolumn{2}{|c|}{ TDS } & \multicolumn{2}{|c|}{$\mathbf{P h}$} & \multicolumn{2}{|c|}{ Turbidity } & \multirow[b]{2}{*}{$\stackrel{\dot{\Xi}}{\ddot{\Xi}}$} & \multirow[b]{2}{*}{$\frac{\mathscr{E}}{\sum}$} \\
\hline 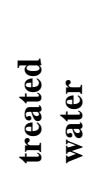 & 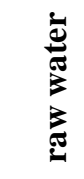 & 冚 & 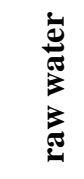 & 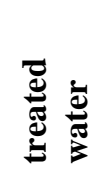 & 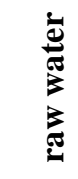 & 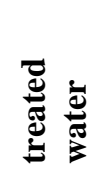 & 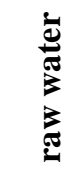 & 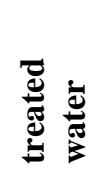 & 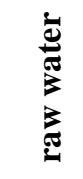 & 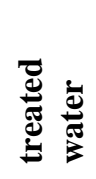 & 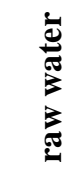 & 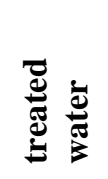 & 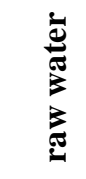 & 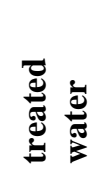 & 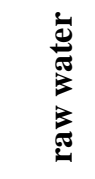 & & \\
\hline 73 & $\overline{83}$ & 1.1 & 0.88 & 95 & 144 & 400 & 650 & 459 & 478 & 393 & 400 & 7.43 & 8.44 & 16 & 5 & 10 & Dec. \\
\hline 74 & 57 & 0.52 & 0.90 & 23 & 27 & 401 & 622 & 492 & 486 & 396 & 421 & 8.2 & 8.1 & 14 & 62 & 9.4 & Jan. \\
\hline 77 & 60 & 1.1 & 1.9 & 19 & 29 & 391 & 597 & 352 & 390 & 460 & 513 & 8 & 7.9 & 9 & 51 & 9.0 & Feb. \\
\hline 76 & 62 & 1 & 0.9 & 18 & 29 & 410 & 570 & 335 & 350 & 450 & 500 & 7.8 & 7.9 & 8.8 & 45 & 20 & March \\
\hline 74 & 53 & 1 & 1.9 & 96 & 640 & 400 & 645 & 422 & 430 & 386 & 393 & 7.7 & 7.8 & 15 & 52 & 20 & April \\
\hline \multirow[t]{2}{*}{75} & 58 & 0.51 & 0.93 & 20 & 25 & 403 & 600 & 450 & 473 & 395 & 400 & 8 & 8 & 12 & 60 & 20 & May \\
\hline & & $\begin{array}{l}200-1 \\
\mathrm{mg} / \mathrm{l}\end{array}$ & & 500 & $\mathrm{ng} / \mathrm{l}$ & 200 & & $\begin{array}{l}500 \\
\text { Mmoh }\end{array}$ & $\mathrm{s} / \mathrm{cm}$ & $1000 \mathrm{~m}$ & $\mathrm{ng} / \mathrm{l}$ & $8.5-6.5$ & & $5 \mathrm{NTL}$ & & 25 & I.S.S \\
\hline
\end{tabular}


Table 9

Chemical and physical tests for Hoth 6 water treatment plant.

\begin{tabular}{|c|c|c|c|c|c|c|c|c|c|c|c|c|c|c|c|c|c|}
\hline \multicolumn{2}{|c|}{ WQI } & \multicolumn{2}{|c|}{$\mathbf{C a}^{+2}$} & \multicolumn{2}{|c|}{ TH } & \multicolumn{2}{|c|}{ Alkali } & \multicolumn{2}{|c|}{ EC } & \multicolumn{2}{|c|}{ TDS } & \multicolumn{2}{|c|}{ PH } & \multicolumn{2}{|c|}{ Turbidity } & \multirow[b]{2}{*}{ 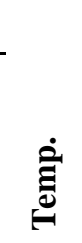 } & \multirow[b]{2}{*}{$\begin{array}{l}\stackrel{n}{E} \\
\stackrel{\Xi}{\Xi} \\
\Xi\end{array}$} \\
\hline 总 & 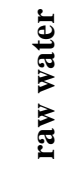 & 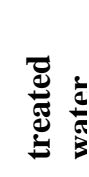 & t) & 离 & 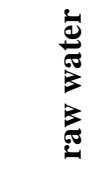 & 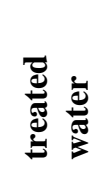 & 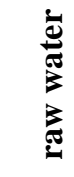 & 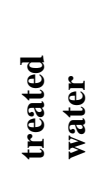 & 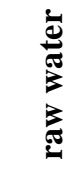 & 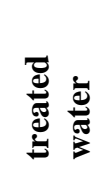 & 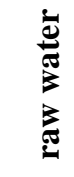 & 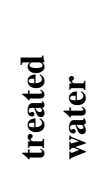 & 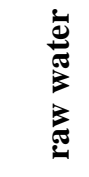 & 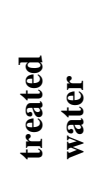 & 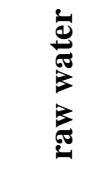 & & \\
\hline 35 & 53 & 0.8 & 0.60 & 37 & 58 & 540 & 675 & 780 & 449 & 344 & 320 & 8.09 & 8.10 & 12 & 1001 & 10 & Dec. \\
\hline 63 & 52 & 0.8 & 0.65 & 37 & 56 & 40 & 680 & 771 & 449 & 343 & 318 & 8.07 & 8.09 & 14 & 1010 & 9.4 & \\
\hline 65 & 53 & 1 & 1.2 & 18 & 19.28 & 495 & 610 & 691 & 429 & 334 & 302 & 9.0 & 9.1 & 10.1 & 981 & 9.0 & Feb. \\
\hline 67 & 53 & 1.1 & 1.7 & 17 & 61 & 403 & 615 & 609 & 500 & 329 & 300 & 8 & 8.1 & 8 & 104 & 20 & March \\
\hline 65 & 49 & 1.1 & 7.9 & 14 & 50 & 620 & 583 & 702 & 600 & 295 & 286 & 7. & 7.8 & 9 & 89 & 20 & April \\
\hline \multirow[t]{2}{*}{65} & 49 & 1 & 7.8 & 12 & 47 & 622 & 580 & 700 & 605 & 299 & 290 & 7.9 & 7.6 & 8.5 & 85 & 20 & May \\
\hline & & $\begin{array}{l}200-1 \\
\mathrm{mg} / 1\end{array}$ & & 500 & & 200 & & $\begin{array}{l}500 \\
\text { Mmoh }\end{array}$ & $\mathrm{s} / \mathrm{cm}$ & 100 & & $8.5-6.5$ & & $5 \mathrm{NTl}$ & & 25 & I.S.S \\
\hline
\end{tabular}

Table 10

Chemical and physical tests for AL Basal water treatment plant.

\begin{tabular}{|c|c|c|c|c|c|c|c|c|c|c|c|c|c|c|c|c|c|}
\hline \multicolumn{2}{|c|}{ WQI } & \multicolumn{2}{|c|}{$\mathrm{Ca}^{+2}$} & \multicolumn{2}{|c|}{ TH } & \multicolumn{2}{|c|}{ Alkali } & \multicolumn{2}{|c|}{ EC } & \multicolumn{2}{|c|}{ TDS } & \multicolumn{2}{|c|}{$\mathbf{P H}$} & \multicolumn{2}{|c|}{ Turbidity } & \multirow[b]{2}{*}{ 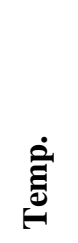 } & \multirow[b]{2}{*}{$\begin{array}{l}\stackrel{n}{E} \\
\stackrel{\Xi}{\Xi}\end{array}$} \\
\hline 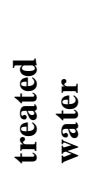 & 氙 & 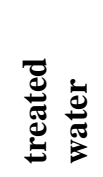 & 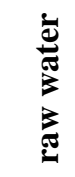 & 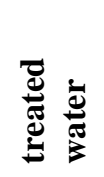 & 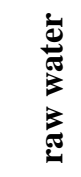 & 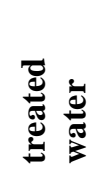 & 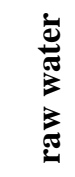 & 苞 & 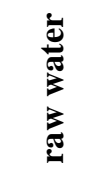 & 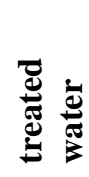 & 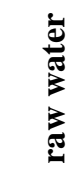 & 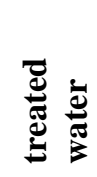 & 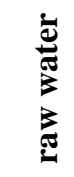 & 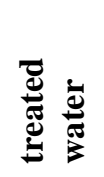 & 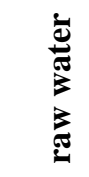 & & \\
\hline 73 & 66 & 33 & 50 & 80 & 87 & 320 & 630 & 323 & 445 & 227 & 331 & 7.5 & 7.1 & 19.4 & 29.7 & 15.1 & Dec. \\
\hline 68 & 45 & 45 & 86 & 98 & 193 & 311 & 591 & 610 & 620 & 295 & 32 & 7.2 & 7. & 0 & 1 & 9.1 & $\mathrm{~J}_{\mathrm{z}}$ \\
\hline 78 & 48 & 45 & 84 & 93 & 120 & 400 & 501 & 498 & 587 & 366 & 495 & 8.1 & 8.2 & 5 & 98 & 20 & Feb. \\
\hline 78 & 54 & 90 & 95 & 61 & 111 & 436 & 509 & 433 & 450 & 450 & 466 & 7.5 & 7.4 & 5 & 90 & 20 & $\begin{array}{l}\text { Marc } \\
\text { h }\end{array}$ \\
\hline 73 & 67 & 33 & 51 & 78 & 80 & 318 & 622 & 320 & 440 & 223 & 334 & 8.2 & 8.2 & 18.7 & 27.3 & 20 & April \\
\hline \multirow[t]{2}{*}{87} & 55 & 75 & 75 & 51 & 110 & 430 & 500 & 430 & 444 & 430 & 450 & 7.3 & 7.5 & 4.8 & 87 & 20 & May \\
\hline & & \multicolumn{2}{|c|}{$\begin{array}{l}200-125 \\
\mathrm{mg} / 1\end{array}$} & \multicolumn{2}{|c|}{$500 \mathrm{mg} / \mathrm{l}$} & \multicolumn{2}{|c|}{$200 \mathrm{mg} / \mathrm{l}$} & \multicolumn{2}{|c|}{$\begin{array}{c}500 \\
\text { Mmohs } / \mathrm{cm}\end{array}$} & \multicolumn{2}{|c|}{$1000 \mathrm{mg} / \mathrm{l}$} & \multicolumn{2}{|c|}{$8.5-6.5$} & \multicolumn{2}{|c|}{$5 \mathrm{NTU}$} & 25 & I.S.S \\
\hline
\end{tabular}

Table 11

Chemical and physical tests for Tal Ali water treatment plant.

\begin{tabular}{|c|c|c|c|c|c|c|c|c|c|c|c|c|c|c|c|c|c|}
\hline \multicolumn{2}{|c|}{ WQI } & \multicolumn{2}{|c|}{$\mathrm{Ca}^{+2}$} & \multicolumn{2}{|c|}{ TH } & \multicolumn{2}{|c|}{ Alkali } & \multicolumn{2}{|c|}{ EC } & \multicolumn{2}{|c|}{ TDS } & \multicolumn{2}{|c|}{$\mathbf{P H}$} & \multicolumn{2}{|c|}{ Turbidity } & \multirow[b]{2}{*}{$\dot{\vec{\Xi}}$} & \multirow[b]{2}{*}{$\begin{array}{l}\mathscr{D} \\
\stackrel{\Xi}{\Xi}\end{array}$} \\
\hline 总 & 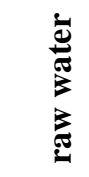 & 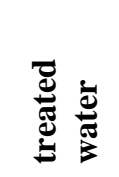 & 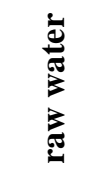 & 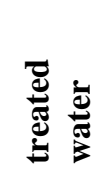 & 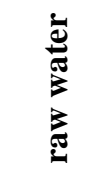 & 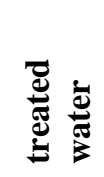 & 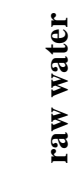 & 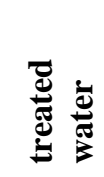 & 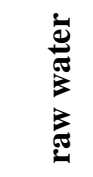 & 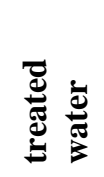 & 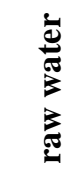 & 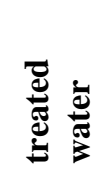 & 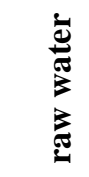 & 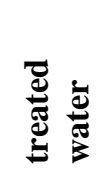 & 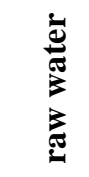 & & \\
\hline 35 & 40 & 1.2 & 1.7 & 48 & 94 & 660 & 890 & 776 & 733 & 530 & 510 & 7 & 7.8 & 32 & 290 & 10 & Dec. \\
\hline 57 & 40 & 1.46 & 1.62 & 53 & 99 & 680 & 900 & 780 & 741 & 543 & 524 & 7.28 & 7.39 & 34.4 & 298 & 9.4 & \\
\hline 64 & 42 & 0.5 & 1.1 & 97.2 & 157 & 660 & 848 & 705 & 663 & 502 & 494 & 7.4 & 7.5 & 10.5 & 205 & 9.0 & Feb. \\
\hline 65 & 48 & 0.9 & 1.5 & 51 & 130 & 593 & 760 & 699 & 610 & 516 & 483 & 8 & 7.8 & 8 & 89 & 20 & $\underset{\mathrm{h}}{\operatorname{Marc}}$ \\
\hline 75 & 51 & 1.2 & 1.9 & 31 & 89 & 601 & 773 & 693 & 640 & 500 & 490 & 8.1 & 8 & 5 & 62 & 20 & April \\
\hline \multirow[t]{2}{*}{75} & 52 & 0.9 & 1.4 & 29 & 81 & 600 & 763 & 650 & 530 & 505 & 495 & 7.2 & 7.9 & 5 & 59 & 20 & May \\
\hline & & \multicolumn{2}{|c|}{$\begin{array}{l}200-125 \\
\mathrm{mg} / \mathrm{l}\end{array}$} & \multicolumn{2}{|c|}{$500 \mathrm{mg} / \mathrm{l}$} & \multicolumn{2}{|c|}{$200 \mathrm{mg} / \mathrm{l}$} & \multicolumn{2}{|c|}{$\begin{array}{l}-500 \\
1 \mathrm{Mmohs} / \mathrm{cm}\end{array}$} & \multicolumn{2}{|c|}{$1000 \mathrm{mg} / \mathrm{l}$} & \multicolumn{2}{|c|}{$8.5-6.5$} & \multicolumn{2}{|c|}{$5 \mathrm{NTU}$} & 25 & I.S.S \\
\hline
\end{tabular}


Table 12

Chemical and physical tests for Al-Shalalah water treatment plant

\begin{tabular}{|c|c|c|c|c|c|c|c|c|c|c|c|c|c|c|c|c|c|}
\hline \multicolumn{2}{|c|}{ WQI } & \multicolumn{2}{|c|}{$\mathrm{Ca}^{+2}$} & \multicolumn{2}{|c|}{ TH } & \multicolumn{2}{|c|}{ Alkali } & \multicolumn{2}{|c|}{ Ec } & \multicolumn{2}{|c|}{ TDS } & \multicolumn{2}{|c|}{$\mathbf{P h}$} & \multicolumn{2}{|c|}{ turbidity } & \multirow[b]{2}{*}{ 宅 } & \multirow[b]{2}{*}{ 号 } \\
\hline 总 & $\begin{array}{l}\bar{\Xi} \\
\bar{N} \\
\bar{E} \\
\bar{E}\end{array}$ & 总 & 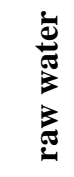 & 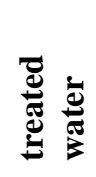 & 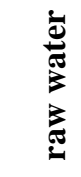 & 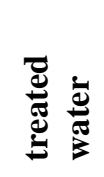 & 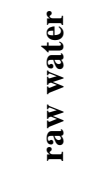 & 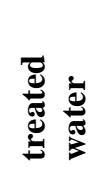 & 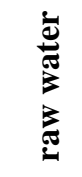 & 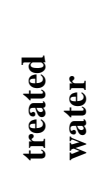 & 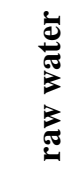 & 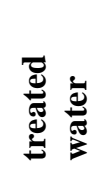 & 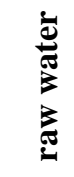 & 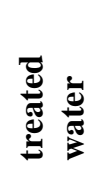 & 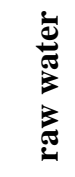 & & \\
\hline 87 & 46 & 0.95 & 1.18 & 37 & 50 & 420 & 587 & 437 & 486 & 280 & 344 & 8.15 & 8.3 & 1.09 & 223 & 10 & Dec. \\
\hline 87 & 46 & 0.94 & 1.19 & 43 & 56 & 420 & 590 & 388 & 485 & 280 & 350 & 8.18 & 8.6 & 1.00 & 225 & 9.4 & Jan. \\
\hline 87 & 46 & 1.1 & 1.9 & 68.2 & 87 & 430 & 570 & 471 & 382 & 401 & 291 & 8.1 & 8.6 & 1.2 & 239 & 9.0 & Feb. \\
\hline 87 & 53 & 1 & 1.8 & 22 & 73 & 435 & 501 & 402 & 391 & 410 & 400 & 8.1 & 8.1 & 0.9 & 101 & 20 & March \\
\hline 87 & 55 & 0.9 & 1.2 & 31 & 91 & 430 & 501 & 459 & 465 & 402 & 329 & 8.1 & 8 & 1 & 83 & 20 & April \\
\hline \multirow[t]{2}{*}{87} & 56 & 0.8 & 1.1 & 28 & 87 & 434 & 502 & 463 & 467 & 405 & 327 & 8.9 & 8.1 & 0.9 & 80 & 20 & May \\
\hline & & \multicolumn{2}{|c|}{$\begin{array}{l}200-125 \\
\mathrm{mg} / \mathrm{l}\end{array}$} & \multicolumn{2}{|c|}{$500 \mathrm{mg} / \mathrm{l}$} & \multicolumn{2}{|c|}{200 mg/l } & \multicolumn{2}{|c|}{$\begin{array}{l}500 \\
\text { Mmohs/cm }\end{array}$} & \multicolumn{2}{|c|}{$1000 \mathrm{mg} / 1$} & \multicolumn{2}{|c|}{$8.5-6.5$} & \multicolumn{2}{|c|}{$5 \mathrm{NTU}$} & 25 & I.S.S \\
\hline
\end{tabular}

Table 13

Chemical and physical tests for Sin Althiban water treatment plant.

\begin{tabular}{|c|c|c|c|c|c|c|c|c|c|c|c|c|c|c|c|c|c|}
\hline \multicolumn{2}{|c|}{ WQI } & \multicolumn{2}{|c|}{$\mathbf{C a}^{+2}$} & \multicolumn{2}{|c|}{ TH } & \multicolumn{2}{|c|}{ Alkali } & \multicolumn{2}{|c|}{ Ec } & \multicolumn{2}{|c|}{ TDS } & \multicolumn{2}{|c|}{ ph } & \multicolumn{2}{|c|}{ Turbidity } & \multirow[b]{2}{*}{$\stackrel{\dot{\vec{\Xi}}}{\stackrel{\vec{\Xi}}{\epsilon}}$} & \multirow[b]{2}{*}{ } \\
\hline 莺 & 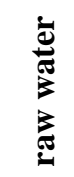 & 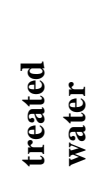 & 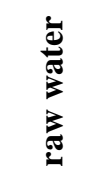 & 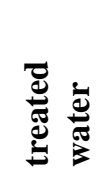 & 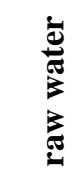 & 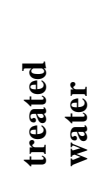 & 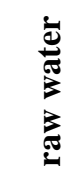 & 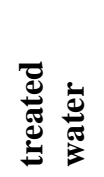 & 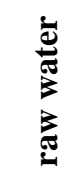 & 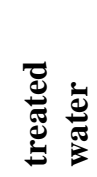 & 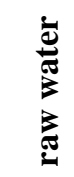 & 总 & 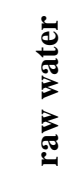 & 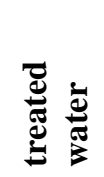 & 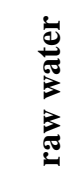 & & \\
\hline 87 & 48 & 0.70 & 0.99 & 42.3 & 50.6 & 440 & 500 & 468 & 490 & 335 & 349 & 8.30 & 7.85 & 3.50 & 180 & 10 & Dec. \\
\hline 86 & 47 & 0.7 & 0.9 & 39.7 & 42.3 & 499 & 532 & 460 & 493 & 382 & 400 & 7.95 & 8.31 & 4.1 & 205 & 9.4 & Jan. \\
\hline 86 & 47 & 1.3 & 1.9 & 23 & 49.1 & 499 & 583 & 500 & 520 & 402 & 415 & 8.1 & 8.1 & 4 & 116 & 9.0 & Feb. \\
\hline 78 & 44 & 1 & 0.84 & 38 & 50 & 440 & 507 & 505 & 430 & 400 & 420 & 7.8 & 8 & 4.8 & 160 & 20 & March \\
\hline 87 & 48 & 0.83 & 0.86 & 43 & 51 & 438 & 498 & 462 & 485 & 328 & 337 & 7.89 & 7.80 & 3.48 & 174 & 20 & April \\
\hline \multirow[t]{2}{*}{86} & 47 & 1 & 1 & 39 & 42 & 490 & 528 & 455 & 488 & 379 & 398 & 8 & 8.22 & 4.3 & 197 & 20 & May \\
\hline & & \multicolumn{2}{|c|}{$\begin{array}{l}200-125 \\
\mathrm{mg} / 1\end{array}$} & \multicolumn{2}{|c|}{$500 \mathrm{mg} / \mathrm{l}$} & \multicolumn{2}{|c|}{$200 \mathrm{mg} / \mathrm{l}$} & \multicolumn{2}{|c|}{$\begin{array}{l}500 \\
\text { Mmohs/cm }\end{array}$} & \multicolumn{2}{|c|}{$1000 \mathrm{mg} / \mathrm{l}$} & \multicolumn{2}{|c|}{$8.5-6.5$} & \multicolumn{2}{|l|}{$5 \mathrm{NTU}$} & 25 & I.S.S \\
\hline
\end{tabular}

Table 14

Chemical and physical tests for Al Shajarah water treatment plant.

\begin{tabular}{|c|c|c|c|c|c|c|c|c|c|c|c|c|c|c|c|c|c|}
\hline \multicolumn{2}{|c|}{ WQI } & \multicolumn{2}{|c|}{$\mathrm{Ca}^{+2}$} & \multicolumn{2}{|c|}{ TH } & \multicolumn{2}{|c|}{ Alkali } & \multicolumn{2}{|c|}{ Ec } & \multicolumn{2}{|c|}{ TDS } & \multicolumn{2}{|c|}{ ph } & \multicolumn{2}{|c|}{ Turbidity } & \multirow[b]{2}{*}{ 宅 } & \multirow[b]{2}{*}{ 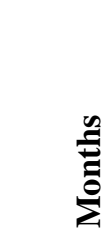 } \\
\hline 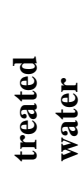 & 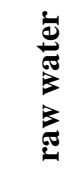 & 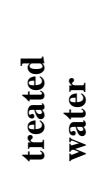 & 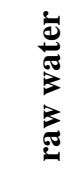 & 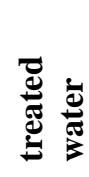 & 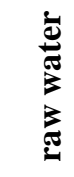 & 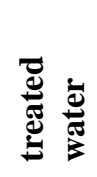 & 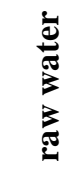 & 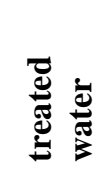 & 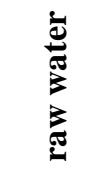 & 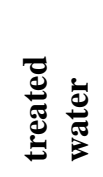 & 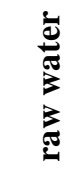 & 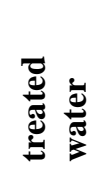 & 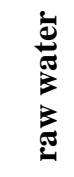 & 冚 & 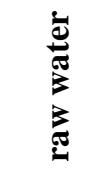 & & \\
\hline 35 & 53 & 9 & 58 & 35 & 55 & 530 & 770 & 563 & 576 & 393 & 414 & 7.7 & 7.6 & 24 & 54.5 & 14 & Dec. \\
\hline 70 & 56 & 10 & 55 & 68 & 78 & 650 & 683 & 420 & 499 & 387 & 400 & 7.5 & 7.5 & 17 & 70 & 9 & Jan. \\
\hline 74 & 54 & 14 & 80 & 41 & 68 & 593 & 621 & 415 & 508 & 390 & 419 & 7.9 & 7.9 & 9 & 53 & 20 & Feb. \\
\hline 76 & 55 & 49 & 60 & 27 & 58 & 583 & 519 & 433 & 502 & 363 & 420 & 7.3 & 7.2 & 6 & 48 & 22 & March \\
\hline 60 & 52 & 6 & 60 & 30 & 45 & 630 & 750 & 550 & 577 & 380 & 414 & 7.2 & 7.6 & 23 & 58 & 12 & April \\
\hline \multirow[t]{2}{*}{76} & 62 & 40 & 85 & 25 & 56 & 581 & 510 & 444 & 500 & 370 & 430 & 7.5 & 7.3 & 6.4 & 45 & 20 & May \\
\hline & & \multicolumn{2}{|c|}{$\begin{array}{l}200-125 \\
\mathrm{mg} / \mathrm{l}\end{array}$} & \multicolumn{2}{|c|}{$500 \mathrm{mg} / \mathrm{l}$} & \multicolumn{2}{|c|}{200 mg/l } & \multicolumn{2}{|c|}{$\begin{array}{l}500 \\
\text { Mmohs/cm }\end{array}$} & \multicolumn{2}{|c|}{$1000 \mathrm{mg} / \mathrm{l}$} & \multicolumn{2}{|c|}{$8.5-6.5$} & \multicolumn{2}{|c|}{$5 \mathrm{NTU}$} & 25 & I.S.S \\
\hline
\end{tabular}


Table 15

Chemical and physical tests for Gharib water treatment plant.

\begin{tabular}{|c|c|c|c|c|c|c|c|c|c|c|c|c|c|c|c|c|c|}
\hline \multicolumn{2}{|c|}{ WQI } & \multicolumn{2}{|c|}{$\mathrm{Ca}^{+2}$} & \multicolumn{2}{|c|}{ TH } & \multicolumn{2}{|c|}{ Alkali } & \multicolumn{2}{|c|}{ EC } & \multicolumn{2}{|c|}{ TDS } & \multicolumn{2}{|c|}{$\mathbf{P h}$} & \multicolumn{2}{|c|}{ Turbidity } & \multirow[b]{2}{*}{ 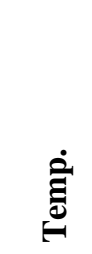 } & \multirow[b]{2}{*}{ 总 } \\
\hline 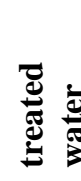 & 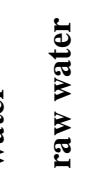 & 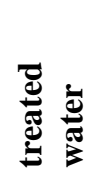 & 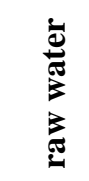 & 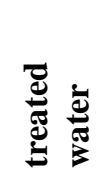 & 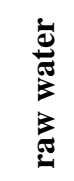 & 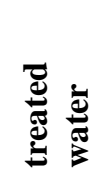 & 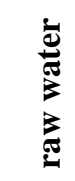 & 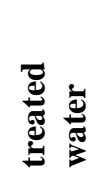 & 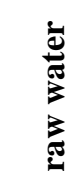 & 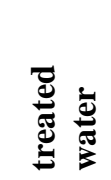 & 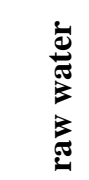 & 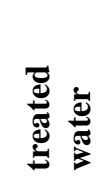 & 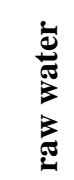 & 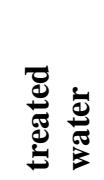 & 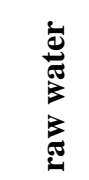 & & \\
\hline$\overline{35}$ & 53 & 1.17 & 0.81 & 88 & 160 & 410 & 770 & 649 & 576 & 463 & 414 & 7.9 & 7.6 & 13 & 54.5 & 10 & Dec. \\
\hline 66 & 50 & 0.82 & 1.5 & 14 & 18 & 403 & 702 & 644 & 564 & 450 & 453 & 7.93 & 8.0 & 12 & 72 & 9.4 & $\mathrm{Ja}$ \\
\hline 68 & 62 & 1.6 & 2 & 17 & 20 & 415 & 639 & 513 & 500 & 425 & 419 & 8.1 & 7.8 & 7 & 41 & 9.0 & Feb. \\
\hline 74 & 54 & 0.82 & 1.8 & 15 & 18 & 420 & 600 & 500 & 520 & 418 & 420 & 8 & 7.6 & 14 & 52 & 20 & March \\
\hline 66 & 53 & 1 & 0.80 & 86 & 158 & 418 & 768 & 647 & 570 & 460 & 410 & 7.7 & 7.8 & 11 & 54 & 20 & April \\
\hline \multirow[t]{2}{*}{66} & 51 & 1 & 1.3 & 12 & 16 & 400 & 698 & 600 & 562 & 445 & 448 & 8 & 8 & 13.5 & 70 & 20 & May \\
\hline & & $\begin{array}{l}200-1 \\
\mathrm{mg} / \mathrm{l}\end{array}$ & & 500 & & 2001 & & $\begin{array}{l}500 \\
\text { Mmoh }\end{array}$ & $\mathrm{s} / \mathrm{cm}$ & $1000 \mathrm{r}$ & & $8.5-6.5$ & & $5 \mathrm{NTU}$ & & 25 & I.S.S \\
\hline
\end{tabular}

Table 16

Chemical and physical tests for Al Tarqia water treatment plant.

\begin{tabular}{|c|c|c|c|c|c|c|c|c|c|c|c|c|c|c|c|c|c|}
\hline \multicolumn{2}{|c|}{ WQI } & \multicolumn{2}{|c|}{$\mathbf{C a}^{+2}$} & \multicolumn{2}{|c|}{ TH } & \multicolumn{2}{|c|}{ Alkali } & \multicolumn{2}{|c|}{ EC } & \multicolumn{2}{|c|}{ TDS } & \multicolumn{2}{|c|}{$\mathbf{P h}$} & \multicolumn{2}{|c|}{ Turbidity } & \multirow[b]{2}{*}{ 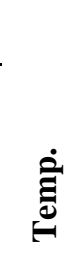 } & \multirow[b]{2}{*}{$\stackrel{n}{E}$} \\
\hline 总 & 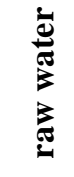 & 总 & 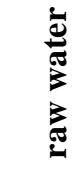 & 冚 & 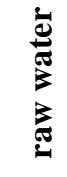 & 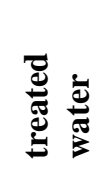 & 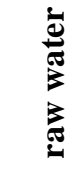 & 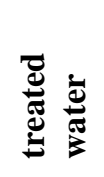 & 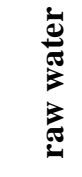 & 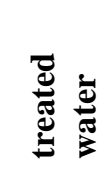 & 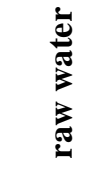 & 兽 & 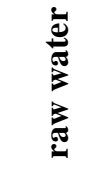 & 总 & 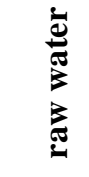 & & \\
\hline 39 & 53 & 50 & 36 & 54 & 72 & 780 & 880 & 548 & 645 & 390 & 465 & 8.08 & 8.05 & 2 & 47 & 14 & Dec. \\
\hline 77 & 53 & 39 & 43 & 52 & 86 & 456 & 676 & 593 & 627 & 389 & 472 & 8.1 & 8.3 & 2.5 & 50 & 9 & $\mathrm{Ja}$ \\
\hline 85 & 54 & 50 & 90 & 48 & 77 & 530 & 600 & 469 & 564 & 4 & 42 & 8 & 7.4 & 1.8 & 49 & 20 & Feh \\
\hline 77 & 56 & 95 & 105 & 29 & 65 & 501 & 597 & 589 & 573 & 393 & 400 & 0 & 8.1 & 1.2 & 43 & 21 & March \\
\hline 74 & 54 & 50 & 35 & 53 & 72 & 750 & 870 & 550 & 640 & 370 & 450 & 7.08 & 8.04 & 1.8 & 42 & 13 & April \\
\hline \multirow[t]{2}{*}{77} & 55 & 100 & 155 & 28 & 55 & 500 & 580 & 570 & 570 & 380 & 390 & 8.4 & 7.1 & 1.2 & 44 & 11 & May \\
\hline & & $\begin{array}{l}200-12 \\
\mathrm{mg} / \mathrm{l}\end{array}$ & & & & $200 n$ & & $\begin{array}{l}500 \\
\text { Mmoh }\end{array}$ & & $1000 \mathrm{~ms}$ & & $8.5-6.5$ & & $5 \mathrm{NTU}$ & & 25 & I.S.S \\
\hline
\end{tabular}

Table 17

Chemical and physical tests for Abbasid water treatment plant.

\begin{tabular}{|c|c|c|c|c|c|c|c|c|c|c|c|c|c|c|c|c|c|}
\hline \multicolumn{2}{|c|}{ WQI } & \multicolumn{2}{|c|}{$\mathrm{Ca}^{+2}$} & \multicolumn{2}{|c|}{ TH } & \multicolumn{2}{|c|}{ Alkali } & \multicolumn{2}{|c|}{ EC } & \multicolumn{2}{|c|}{ TDS } & \multicolumn{2}{|c|}{$\mathbf{P H}$} & \multicolumn{2}{|c|}{ Turbidity } & \multirow[b]{2}{*}{ 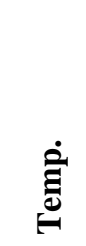 } & \multirow[b]{2}{*}{$\stackrel{\text { Ẽ }}{\sum_{0}^{0}}$} \\
\hline 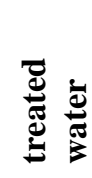 & 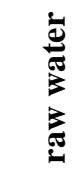 & 胥 & 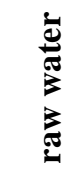 & 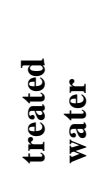 & 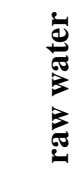 & 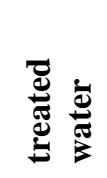 & 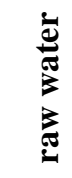 & 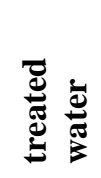 & 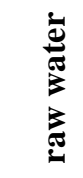 & 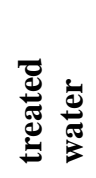 & 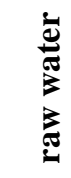 & 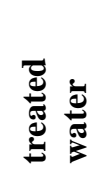 & 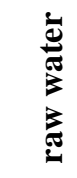 & 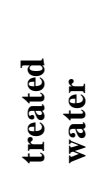 & 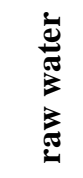 & & \\
\hline 86 & 61 & 0.85 & 1.16 & 32 & 79 & 509 & 670 & 499 & 295 & 440 & 250 & 7.3 & 8.9 & 0.8 & 46 & 10 & Dec. \\
\hline 72 & 53 & 0.9 & 1.19 & 52 & 83 & 520 & 680 & 508 & 306 & 508 & 207 & 87.4 & 8.14 & 15.4 & 52.1 & 9.4 & Jan. \\
\hline 86 & 57 & 0.85 & 1.25 & 76.1 & 91.6 & 498 & 633 & 333 & 291 & 390 & 325 & 7.9 & 8.3 & 1.9 & 66 & 9.0 & Feb. \\
\hline 87 & 61 & 1.15 & 1.39 & 43 & 95 & 442 & 562 & 301 & 250 & 490 & 402 & 7.9 & 7.8 & 1.2 & 50 & 20 & March \\
\hline 85 & 61 & 1 & 1.16 & 37 & 81 & 530 & 620 & 326 & 305 & 433 & 333 & 7.8 & 7.8 & 0.9 & 48 & 20 & April \\
\hline \multirow[t]{2}{*}{86} & 60 & 0.99 & 1.13 & 29 & 72 & 520 & 610 & 329 & 310 & 432 & 330 & 7.4 & 8.1 & 1.4 & 51 & 20 & May \\
\hline & & $\begin{array}{l}200-1 \\
\mathrm{mg} / \mathrm{l}\end{array}$ & & 500 & $\mathrm{hg} / 1$ & 200 & & $\begin{array}{l}500 \\
\text { Mmoh }\end{array}$ & $\mathrm{ns} / \mathrm{cm}$ & $1000 \mathrm{~m}$ & $\mathrm{ng} / \mathrm{l}$ & $8.5-6.5$ & & $5 \mathrm{NTU}$ & & 25 & I.S.S \\
\hline
\end{tabular}


Table 18

Chemical and physical tests for Abu al-Jess water treatment plant.

\begin{tabular}{|c|c|c|c|c|c|c|c|c|c|c|c|c|c|c|c|c|c|}
\hline \multicolumn{2}{|c|}{ WQI } & \multicolumn{2}{|c|}{$\mathrm{Ca}+2$} & \multicolumn{2}{|c|}{ TH } & \multicolumn{2}{|c|}{ Alkali } & \multicolumn{2}{|c|}{$\mathbf{E C}$} & \multicolumn{2}{|c|}{ TDS } & \multicolumn{2}{|c|}{ PH } & \multicolumn{2}{|c|}{ Turbidity } & \multirow[b]{2}{*}{ 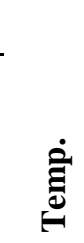 } & \multirow[b]{2}{*}{$\sum_{\Sigma}^{2}$} \\
\hline 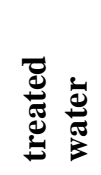 & 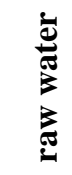 & 总 & 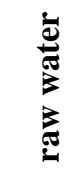 & 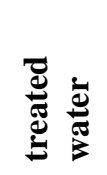 & 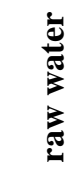 & 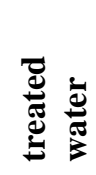 & 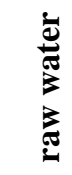 & 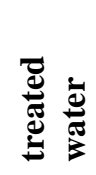 & 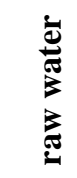 & 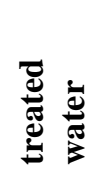 & 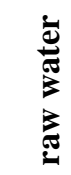 & 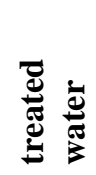 & 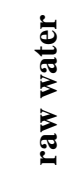 & 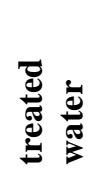 & 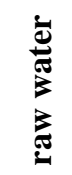 & & \\
\hline$\overline{79}$ & 70 & 0.23 & 0.29 & 20 & 23 & 230 & 640 & 300 & 310 & 222 & 240 & 77 & 8.8 & 5.6 & 18 & 10 & Dec. \\
\hline 79 & 67 & 0.36 & 0.27 & 22 & 25 & 270 & 670 & 325 & 329 & 221 & 234 & 8.8 & 8.7 & 6.30 & 20 & 9.4 & Jan. \\
\hline 76 & 53 & 0.18 & 0.36 & 11.28 & 12.6 & 405 & 701 & 317 & 338 & 535 & 555 & 8.7 & 8.7 & 9 & 100 & 9.0 & Feb. \\
\hline 78 & 55 & 0.14 & 0.30 & 12 & 39 & 410 & 635 & 299 & 320 & 400 & 450 & 8 & 8 & 6 & 82 & 20 & March \\
\hline 76 & 59 & 0.9 & 1.9 & 20 & 31 & 550 & 630 & 289 & 315 & 387 & 443 & 8 & 8.1 & 5.5 & 58 & 20 & April \\
\hline \multirow[t]{2}{*}{85} & 61 & 0.4 & 1.4 & 26 & 29 & 530 & 615 & 287 & 311 & 381 & 439 & 7.1 & 7.9 & 5 & 45 & 20 & May \\
\hline & & $\begin{array}{l}200-12 \\
\mathrm{mg} / \mathrm{l}\end{array}$ & & 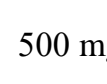 & & 200 & & $\begin{array}{l}500 \\
\text { Mmoh }\end{array}$ & $\mathrm{s} / \mathrm{cm}$ & 100 & & .5 & & 4 & & 25 & I.S.S \\
\hline
\end{tabular}

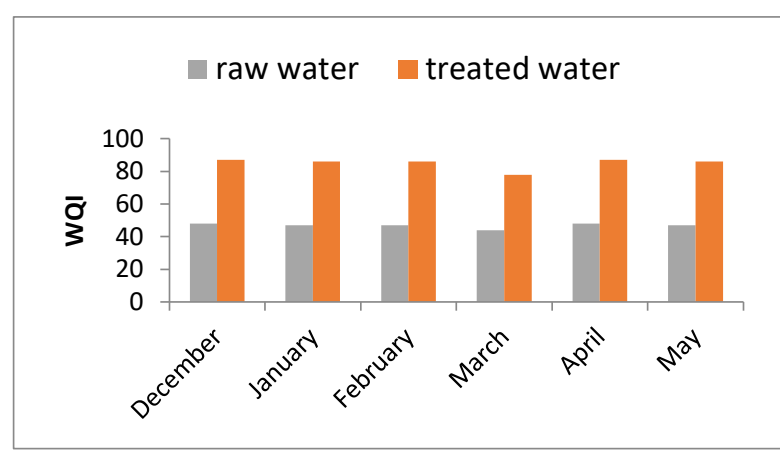

Fig. 11. Water quality index Sin Althiban water treatment plant.

Fig. 12 shows the water quality of Al Shajarah water treatment plant, which shows that the raw water was in the category of the fourth (bad), but after treatment became water in the third category (moderate) except for the month of December has remained water quality in the fourth category as is clear in the Table 14.

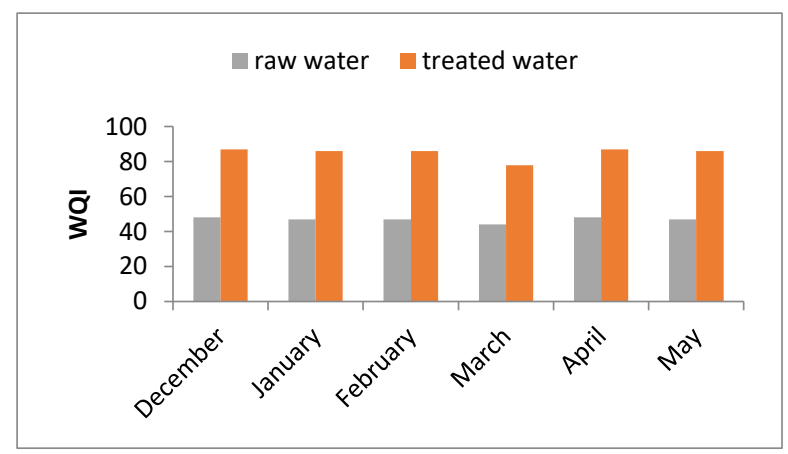

Fig. 12. Water quality index Al Shajarah water treatment plant.

The quality of raw water in (Gharib) water treatment plant was classified as four category (bad) during the study period (Fig. 13). After treatment, the water quality index improved to the third category (moderate) except for December. In the fifth category (very bad), which indicates a defect in this period in the plant as shown in Table 15 .

Fig. 14 shows the results of water quality index for (Al Tarqia) water treatment plant and shows that there was a defect during the month of December where the quality of water treated in category $\mathrm{V}$ (very bad) The rest of the months of the study was in the

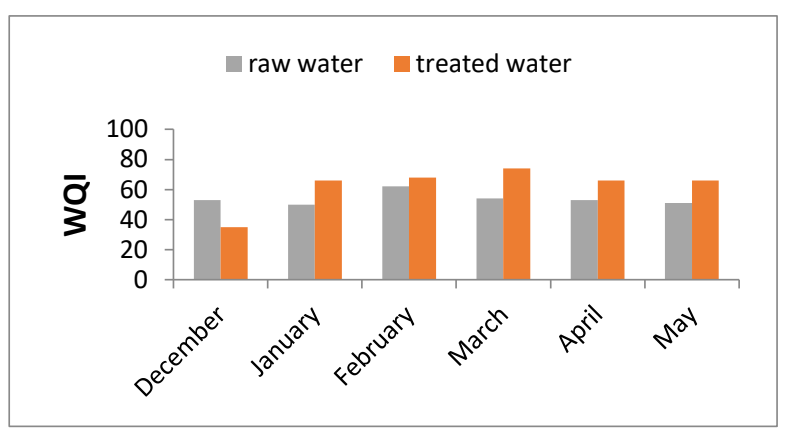

Fig. 13. Water quality index Gharib water treatment plant. second category (good) for the month of Jan, February, March and in the third category (moderate) for April and May as shown in Table 16.

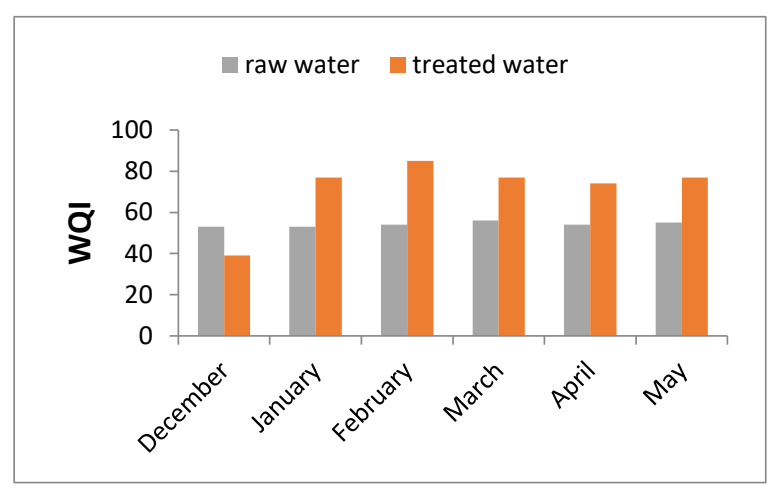

Fig. 14. Water quality index $\mathrm{Al}$ Tarqia water treatment plant.

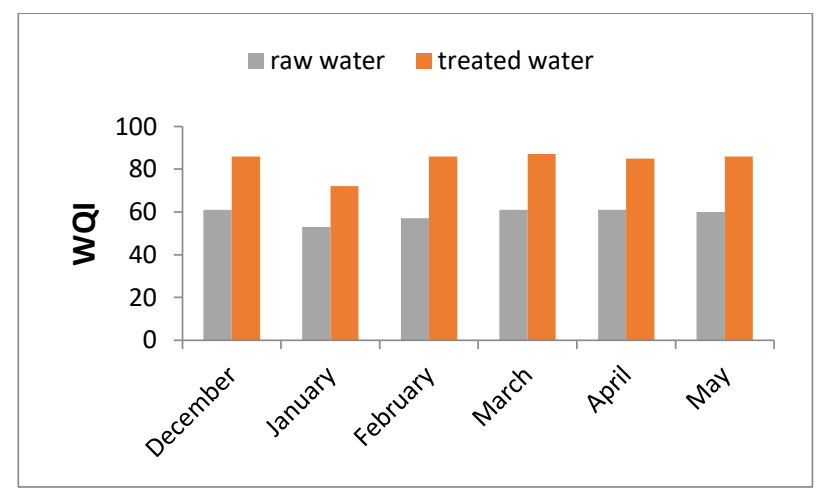

Fig. 15. Water quality index Abbasid water treatment plant. 
Fig. 15 represented Water quality index for (Abbasid) water treatment plant The results indicated that the raw water was classified as category 4 (bad), the treated water was classified in the second category (good) except for Jan. was the quality of water in the third category (moderate) Table 17.

Fig. 16, which represents the water quality index at (Abu al-Jess) water treatment plant indicated that the raw water was in the category of the fourth (bad) to be after treatment in the third category (moderate) except May was in the second category (good) Table 18.

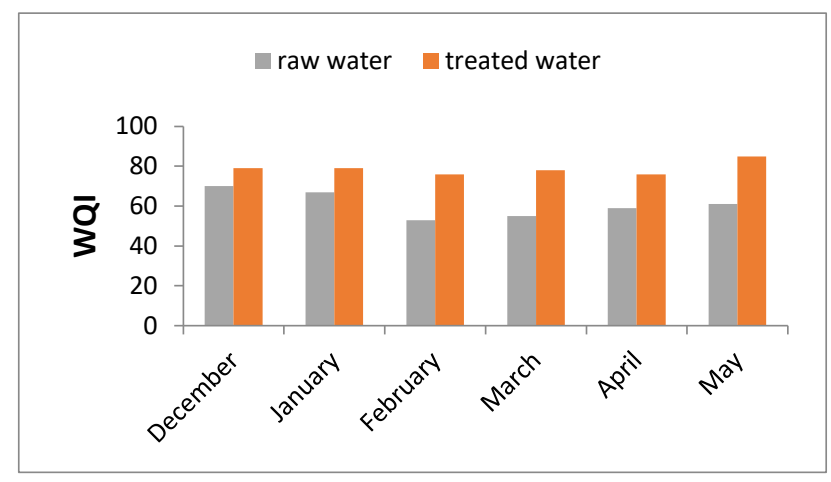

Fig. 16. Water quality index Abu al-Jess water treatment plant.

\section{CONCLUSIONS}

1. The water quality index of raw water was less than (65) for most plants and most of the months of the study, which is classified in the fourth category (bad).

2. The quality of treated water in Al-Musanaa water treatment plant did not change after the treatment and remained in the fourth category, indicating that there is a defect in the treatment of water in this plant.

3. The values of the water quality index for Al-Shalala and Al-Zaban plants ranged from (80-87), which is classified as category II (good), indicating that these plants work well.

4. The water quality index for the rest of the plants was fluctuated between the fourth and third and the second as shown in the tables and shapes.

\section{REFERENCES}

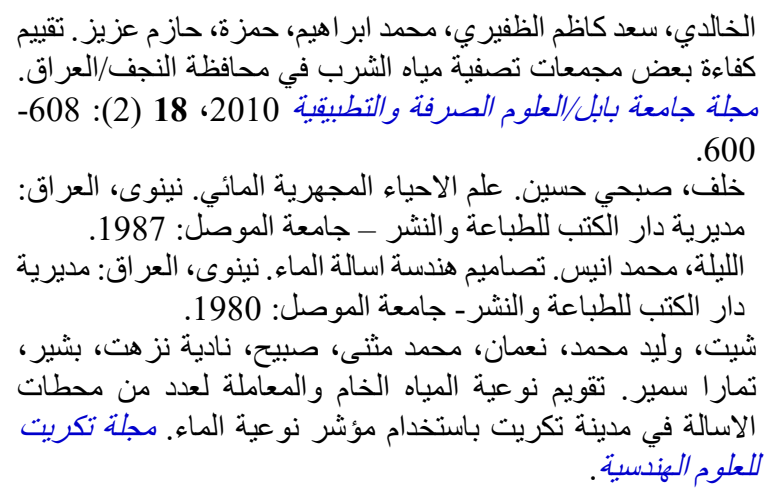

[1]

[5] Muhammed ZB. Evaluation the quality of raw and treated water for number of water treatment plants in Baghdad using water quality index. Engineering and Technology Journal 2015; 33 (6): 1429-1441.

[6] Abdul-Rahman E, Ahmed MF. Comparison of water quality index at intakes of water treatment plants in
Baghdad city. Tikrit Journal of Engineering Sciences 2013; 20 (4):23-34.

[7] Alhadithi, M. Water wells quality assessment for drinking purposes using water Quality index and correlation study in Al-Qaim city. Al-Anbar, Iraq. AlAnbar Journal of Agricultural Sciences 2016; 1:3e$14 \mathrm{e}$.

[8] Al-Alwani NH, Yassin RA. Identification of potable water using water quality index technique in the city of Al-Zanatn, Libya. Iraqi Bulletin of Geology and Mining 2016;12 (3):43-52.

[9] Nua'man MM. Using of index approach to assess water of tigris river as a source to water treatment plants in Salah-Aldin Province, Iraq. Tikrit Journal of Engineering Sciences 2013; 20 (3):53-63.

[10] Al-Bassam BF, Taher RK. Evaluation of shallow groundwater quality in Zehengzhou area (China) using water quality index model. Diyala Journal of Engineering Sciences 2015: 37-45.

[11] Al-Badran F. Determination of water quality index and suitability of Shatt Al Arab River and treated water for some water treated plants in Basrah. Basrah Journal for Engineering Science 2013; 13 (1): 50-62.

[12] APHA, American Public Health Association. Standard methods for the examination of water and wastewater. 20th.ed.33: 1990.

[13] الجهاز المركزي للتقييس والسيطرة النوعية، جمهورية العراق. ICS:13.060.20،2001/417/ .I.S.S 